\title{
WHO Programme on Mental Health: Behavioural Science Learning Modules
}

The World Health Organization has produced a number of Learning Modules, aimed primarily at the education of physicians and medical students, which deal with aspects of communication and psychological treatment for common medical problems. Copies may be obtained from the Division of Mental Health, World Health Organization, 1211 Geneva 27, Switzerland. 